\title{
Erratum
}

\section{Théorème de Torelli pour les cubiques de $\mathbb{P}^{5}$}

\section{Claire Voisin}

IHÉS, 35 route de Chartres, 91440 Bures-sur-Yvette, France (e-mail: voisin@ihes.fr)

Oblatum 9-X-2007 \& 24-I-2008

Published online: 9 February 2008 - (c) Springer-Verlag 2008

Erratum to: Invent. math. 86, 577-601 (1986)

DOI: $10.1007 / \mathrm{BF} 01389270$

Soit $L \cong H^{4}\left(X_{\text {gen }}, \mathbb{Z}\right)$ où $X_{\text {gen }}$ est une hypersurface cubique lisse de $\mathbb{P}^{5}$. $L$ est muni de sa forme d'intersection et de la classe $h^{2}, h=c_{1}\left(\mathcal{O}_{X_{\text {gen }}}(1)\right)$, qui est marquée. Soit $\Gamma:=\operatorname{Aut}\left(L,\langle\rangle,, h^{2}\right)$ et soit $\mathscr{D}$ le domaine des périodes correspondant. Les hypersurfaces cubiques de $\mathbb{P}^{5}$ ayant au plus des points doubles ordinaires étant stables pour l'action de $P G l(6)$, elles sont paramétrées par un espace de modules $\mathcal{M}$, quotient d'un ouvert de $\mathbb{P}\left(H^{0}\left(\mathbb{P}^{5}, \mathcal{O}_{\mathbb{P}^{5}}(3)\right)\right)$ par $P G l(6)$. On sait (voir plus loin) que l'application des périodes, qui à une cubique lisse $X$ associe la structure de Hodge polarisée et marquée sur $H^{4}(X, \mathbb{Z})$, s'étend à $\mathcal{M}$. Le résultat principal de [7] est le suivant :

Théorème 1 L'application des périodes $\mathcal{P}: \mathcal{M} \rightarrow \mathscr{D} / \Gamma$ est un plongement ouvert.

Ce théorème a été redémontré récemment par Looijenga [4].

La stratégie de la démonstration de [7] consiste à étudier l'hypersurface $\mathscr{H} \subset \mathcal{M}$ paramétrant les hypersurfaces contenant un plan. Les deux premières étapes de la démonstration fournissent les énoncés suivants :

Théorème 2 i) La restriction de $\mathcal{P}$ à $\mathscr{H}$ est injective en codimension 1. ii) On a $\mathcal{P}^{-1}(\overline{\mathcal{P}(\mathscr{H})})=\mathscr{H}$.

On concluait alors en utilisant l'amplitude de $\mathscr{H}$ et le fait que l'application des périodes locales est non ramifiée en codimension 1. Pour l'application des périodes locales définie sur l'ouvert de $\mathcal{M}$ paramétrant les cubiques lisses, ce résultat est montré dans [1]. Le résultat s'étend sans difficulté à $\mathcal{M}$ (cf. [7]). 
K. O’Grady a observé que l'argument final de [7] est insuffisant, du fait de la non-propreté de l'application des périodes. On remplace ici cet argument par un argument de même nature, mais qui utilise une estimation sur la codimension du lieu d'indétermination de l'application des périodes. Celle-ci est basée sur le résultat suivant dû à Laza [3]. Notons $\overline{\mathcal{M}}=\mathbb{P}\left(H^{0}\left(\mathbb{P}^{5}, \mathcal{O}_{\mathbb{P}^{5}}(3)\right)\right) / / P G l(6)$ la compactification de $\mathcal{M}$ fournie par la théorie des invariants géométriques de Mumford [6].

Théorème 3 (Laza) i) Les hypersurfaces cubiques à singularités isolées, stables pour l'action de PGl(6), sont les cubiques à singularités isolées simples.

ii) Soit $\mathcal{M}^{\prime} \subset \overline{\mathcal{M}}$ le quotient de cet ensemble par PGl(6). Alors le complément $Z:=\overline{\mathcal{M}} \backslash \mathcal{M}^{\prime}$ est de dimension $\leq 3$.

Nous n'utiliserons en fait qu'une estimation beaucoup plus faible sur la dimension de $Z$, à savoir $\operatorname{dim} Z \leq 8$.

Nous utiliserons de façon cruciale le fait que $\mathcal{P}$ s'étend à $\mathcal{M}^{\prime}$ tout en restant à valeurs dans $\mathscr{D} / \Gamma$ et que de plus, elle est un isomorphisme local au niveau des déformations miniverselles. En d'autres termes, soit $X_{t}$ une hypersurface cubique à singularités simples, et $\mathcal{M}_{t, l o c}$ la base de la déformation universelle de $X_{t}$, (de sorte qu'un voisinage de $t$ dans $\mathcal{M}^{\prime}$ est obtenu comme un quotient de $\mathcal{M}_{t, \text { loc }}$ par le groupe fini des automorphismes de $\left.X_{t}\right)$; soit $\Gamma_{t}$ le groupe de monodromie local (fini) agissant sur $H^{4}\left(X_{0}, \mathbb{Z}\right)$, où $X_{0}$ est une fibre lisse proche de $X_{t}$; alors l'application des périodes locales $\mathcal{P}_{\text {loc }}: \mathcal{M}_{t, l o c} \backslash \delta \rightarrow \mathcal{D} / \Gamma_{t}$, où $\&$ est l'hypersurface discriminante, s'étend en un morphisme sur $\mathcal{M}_{t, \text { loc }}$ (cf. [2]). Notant que $\mathcal{M}_{t, l o c}$ et $\mathcal{D} / \Gamma_{t}$ sont lisses (pour $\mathcal{D} / \Gamma_{t}$, voir [5, p. 182]), le fait que $\mathcal{P}_{\text {loc }}$ n' ait pas de diviseur de ramification sur $\mathcal{M}$ (et donc sur $\mathcal{M}^{\prime}$, car codim $\mathcal{M}^{\prime} \backslash \mathcal{M}>1$ ) entraîne que $\mathcal{P}_{\text {loc }}$ est un isomorphisme local.

Ceci entraîne que l'application $\mathcal{P}$ étendue à $\mathcal{M}^{\prime}$ est ouverte, localement finie et est un isomorphisme local au point $t$ si et seulement si elle est de degré local 1 au point $t$. Montrons maintenant :

Proposition 4 Notant $\mathscr{H}^{\prime}$ la clôture de $\mathscr{H}$ dans $\mathcal{M}^{\prime}$, les énoncés i) et ii) du théorème 2 restent vrais pour $\mathscr{H}^{\prime}$. De plus $\mathcal{P}$ est un isomorphisme local le long de $\mathscr{H}^{\prime}$.

Démonstration. En effet, ii) est satisfait dans $\mathcal{M}$ et donc en codimension 1 sur $\mathcal{M}^{\prime}$. Or $\mathcal{P}$ est localement finie sur $\mathcal{M}^{\prime}$ et donc $\mathcal{P}^{-1}\left(\mathcal{P}\left(\mathscr{H}^{\prime}\right)\right)$ est de codimension pure 1 . Pour i), l'allure locale de $\mathcal{P}$ montre que si $x \neq y \in \mathscr{H}^{\prime}$ satisfont $\mathcal{P}(x)=\mathscr{P}(y)$, de telles paires existent en codimension 1 dans $\mathscr{H}^{\prime}$, et donc dans $\mathscr{H}$, ce qui contredit le fait que i) est vrai en codimension 1 sur $\mathscr{H} \subset \mathscr{H}^{\prime}$ comme montré dans [7] (cet argument est dû à K. O'Grady). Le dernier énoncé résulte alors du fait que $\mathscr{P}$ est non ramifiée génériquement le long de $\mathscr{H}^{\prime}$, de sorte que par ii), le degré local de $\mathcal{P}$ le long de $\mathscr{H}^{\prime}$ est égal au degré local de $\mathcal{P}_{\mid \mathscr{H}^{\prime}}$, c'est-à-dire 1 d'après i). 
Conclusion de la preuve du théorème 1 . On veut montrer l'injectivité de $\mathcal{P}$ sur $\mathcal{M}$. Supposons qu'on ait $x \neq y \in \mathcal{M}$ avec $\mathcal{P}(x)=\mathcal{P}(y)$. Comme $\mathcal{P}$ est ouverte sur $\mathcal{M}^{\prime}$, on peut supposer que $\mathcal{P}(x) \in \mathscr{D} / \Gamma$ paramètre une structure de Hodge polarisée (avec la classe $h^{2}$ marquée) sans automorphisme non trivial. Par ce qui précède, ceci garantit que l'application $\mathcal{P}$ est un isomorphisme local au voisinage de $x$ et de $y$. Soit $K \subset \overline{\mathcal{M}}$ l'intersection complète de 9 hypersurfaces suffisamment amples et suffisamment génériques contenant $x$ et $y$. Comme $\operatorname{dim} Z \leq 8, K$ ne rencontre pas $Z$ et donc $\mathcal{P}$ est bien définie le long de $K$. De plus $K$ est projective (de dimension 11).

Comme $K$ est une intersection complète de 9 hypersurfaces suffisamment générales, il en va de même de $\mathcal{P}(K)$ au voisinage de $\mathcal{P}(x)=\mathcal{P}(y)$. Notant $K_{x}$ et $K_{y}$ des voisinages de $x$ et $y$ dans $K$, les surfaces $S_{x} \subset K$ et $S_{y} \subset K$ définies respectivement comme $K_{x} \cap \mathcal{P}^{-1}\left(\mathcal{P}\left(K_{y}\right)\right)$ au voisinage de $x$, et $K_{y} \cap \mathcal{P}^{-1}\left(\mathcal{P}\left(K_{x}\right)\right)$ au voisinage de $y$ s'envoient par $\mathcal{P}$ sur la même surface $\mathcal{P}\left(K_{x}\right) \cap \mathcal{P}\left(K_{y}\right)$. Soit $\Sigma \subset K$ la clôture de Zariski de $S_{x} \cup S_{y}$ dans $K$. Ce qui précède montre que $\Sigma$ est une surface projective et que $\mathcal{P}_{\mid \Sigma}$ est de degré au moins deux au-dessus de son image $\Sigma^{\prime}$, (qui est irréductible).

Notons maintenant que l'hypersurface $\overline{\mathscr{H}}$ est ample dans $\overline{\mathcal{M}}$. Il en résulte que $\Sigma \cap \overline{\mathscr{H}} \neq \emptyset$, et comme $\Sigma \subset K \subset \mathcal{M}^{\prime}$, on a en fait $\Sigma \cap \mathscr{H}^{\prime} \neq \emptyset$. Mais comme $\mathcal{P}$ est un isomorphisme local au voisinage de $\mathcal{H}^{\prime}$ et $\mathcal{P}$ est injective sur $\mathscr{H}^{\prime}$, la restriction $\mathcal{P}_{\mid \Sigma}$ de $\mathcal{P}$ à $\Sigma$ est un plongement sur un voisinage de $\Sigma \cap \mathscr{H}^{\prime}$. Par ailleurs, on a aussi

$$
\mathcal{P}_{\mid \Sigma}^{-1}\left(\mathcal{P}_{\mid \Sigma}\left(\Sigma \cap \mathscr{H}^{\prime}\right)\right)=\Sigma \cap \mathscr{H}^{\prime}
$$

par la proposition 4. Il en résulte que pour un voisinage $V$ de $\mathcal{P}_{\mid \Sigma}\left(\Sigma \cap \mathscr{H}^{\prime}\right)$ dans $\Sigma^{\prime}, \mathcal{P}_{\mid \Sigma}$ est un isomorphisme de $\mathcal{P}_{\mid \Sigma}^{-1}(V)$ sur $V$, ce qui contredit le fait que $\mathcal{P}_{\mid \Sigma}$ est de degré $\geq 2$ sur son image $\Sigma^{\prime}$.

Remarque 5 Il est peut-être possible de remplacer dans cette preuve l'hypersurface $\mathscr{H}$ par l'hypersurface discriminante $\$$. Pour cette dernière, l'analogue du théorème 2 est beaucoup plus facile à démontrer, à l'exception peut-être de la condition "en codimension 1" du i). Cela fournirait une preuve très simplifiée du théorème 1 .

Remerciements. Je remercie Kieran O'Grady pour m'avoir signalé cette erreur et pour de nombreux échanges à ce sujet.

\section{References}

1. Beauville, A., Donagi, R.: La variété des droites d'une hypersurface cubique de dimension 4. C. R. Acad. Sci., Paris, Sér. I, Math. 301(14), 703-706 (1985)

2. Griffiths, P.A.: Periods of integrals on algebraic manifolds. III. Some global differentialgeometric properties of the period mapping. Publ. Math., Inst. Hautes Étud. Sci. 38, 125-180 (1970)

3. Laza, R.: The moduli space of cubic fourfolds. À paraître au J. Algebr. Geom. (arXiv: 0704.3256) 
4. Looijenga, E.: The period map for cubic fourfolds. (arXiv: 0705.0951)

5. Looijenga, E.: Isolated Singular Points on Complete Intersections. Lond. Math. Soc. Lect. Note Ser., vol. 77. Cambridge University Press, Cambridge (1984)

6. Mumford, D., Fogarty, J., Kirwan, F.: Geometric Invariant Theory, 3rd edn. Ergeb. Math. Grenzgeb. (2), vol. 34. Springer, Berlin (1994)

7. Voisin, C.: Théorème de Torelli pour les cubiques de $\mathbb{P}^{5}$. Invent. Math. 86, 577-601 (1986) 